\title{
Altered Cerebrospinal Fluid Exosomal microRNA Levels in Young-Onset Alzheimer's Disease and Frontotemporal Dementia
}

\author{
Yi Jayne Tan ${ }^{\mathrm{a}}$, Benjamin Y.X. Wong ${ }^{\mathrm{a}}$, Ramanathan Vaidyanathan ${ }^{\mathrm{b}}$, Sivaramapanicker Sreejith ${ }^{\mathrm{c}}$, \\ Sook Yoong Chia ${ }^{\mathrm{d}}$, Nagaendran Kandiah ${ }^{\mathrm{a}, \mathrm{e}, \mathrm{f}}$, Adeline S.L. Ng ${ }^{\mathrm{a}, \mathrm{e}, 1}$ and Li Zeng ${ }^{\mathrm{d}, \mathrm{e}, \mathrm{f}, *, 1}$ \\ ${ }^{a}$ Department of Neurology, National Neuroscience Institute, Tan Tock Seng Hospital, Singapore \\ ${ }^{\mathrm{b}}$ Department of Biomedical Engineering, National University of Singapore, Singapore \\ ${ }^{\mathrm{c}}$ Biomedical Institute for Global Health Research \& Technology (BIGHEART), National University of Singapore, \\ Singapore \\ ${ }^{\mathrm{d}}$ Neural Stem Cell Research Lab, Department of Research, National Neuroscience Institute, Singapore \\ ${ }^{\mathrm{e}}$ Neuroscience and Behavioural Disorders Unit, Duke-NUS Medical School, Singapore \\ ${ }^{\mathrm{f}}$ Lee Kong Chian School of Medicine, Nanyang Technology University, Novena Campus, Singapore
}

Accepted 15 September 2021

Pre-press 10 October 2021

Published 28 October 2021

\begin{abstract}
.
Background: micro-RNAs (miRNAs) are stable, small, non-coding RNAs enriched in exosomes. Their variation in levels according to different disease etiologies have made them a promising diagnostic biomarker for neurodegenerative diseases such as Alzheimer's disease (AD). Altered expression of miR-320a, miR-328-3p, and miR-204-5p have been reported in AD and frontotemporal dementia (FTD).

Objective: To determine their reliability, we aimed to examine the expression of three exosomal miRNAs isolated from cerebrospinal fluid (CSF) of patients with young-onset AD and FTD ( $<65$ years), correlating with core AD biomarkers and cognitive scores.

Methods: Exosomes were first isolated from CSF samples of 48 subjects ( 8 controls, 28 AD, and 12 FTD), followed by RNA extraction and quantitative PCR to measure the expression of miR-320a, miR-328-3p, and miR-204-5p.

Results: Expression of all three markers (miR-320a $(p=0.005)$, miR-328-3p $(p=0.049)$, and miR-204-5p $(p=0.036))$ were significantly lower in AD versus controls. miR-320a was reduced in FTD versus controls $(p=0.049)$ and miR-328-3p was lower in AD versus FTD $(p=0.054)$. Notably, lower miR-328-3p levels could differentiate AD from FTD and controls with an AUC of 0.702, 95\% CI: 0.534-0.870, and showed significant correlation with lower CSF A $\beta_{42}$ levels $(r=0.359, p=0.029)$. Pathway enrichment analysis identified potential targets of miR-328-3p implicated in the AMPK signaling pathway linked to amyloid- $\beta$ and tau metabolism in AD.
\end{abstract}

\footnotetext{
${ }^{1}$ Joint senior author.

${ }^{*}$ Correspondence to: Dr. Li Zeng, Department of Research (Level 6), National Neuroscience Institute, 11 Jalan Tan Tock Seng, Singapore 308433. E-mail: Li_Zeng@nni.com.sg.
} 
Conclusion: Overall, we demonstrated miR-320a and miR-204-5p as reliable biomarkers for AD and FTD and report miR-328-3p as a novel AD biomarker.

Keywords: Alzheimer's disease, biomarker, cerebrospinal fluid, exosome, frontotemporal dementia, miRNAs, young-onset Alzheimer's disease

\section{INTRODUCTION}

micro-RNAs (miRNAs) are stable, small, non-coding RNAs (composed of 21-23 nucleotides) involved in the degradation and/or translational repression of target messenger RNAs (mRNAs) [1]. They also have various roles in cell development, differentiation, proliferation, apoptosis, synaptic plasticity, and memory [2].

Cell-to-cell communication is considered a key role for miRNAs as they are released into the extracellular milieu by several mechanisms and packed into small extracellular vesicles such as exosomes, for exchange of genetic material between cells [3]. Exosomes are small membrane-enclosed vesicles released by most cell types to carry out specific cellcell communications [4] by influencing gene expression and protein activity in recipient cells. miRNAs are enriched in exosomes, and their modifications, as well as variation in levels according to different disease etiologies have made them a promising diagnostic biomarker candidate for neurodegenerative diseases, particularly in Alzheimer's disease (AD) [5-8] and frontotemporal dementia (FTD) [9, 10]. Compared to circulating and intracellular miRNAs, miRNA encapsulated in exosome is reportedly to be more stable in biological fluids [11, 12].

In $\mathrm{AD}$, neuronal exosomes may be involved in the release of $A \beta_{42}$ due to early endosomal maturation [13], but may also play a role in sequestering intracerebral $A \beta_{42}$ [14]. Of note, miR-328 [15] was shown in an $\mathrm{AD}$ mouse model to recognize specific binding sites in the $3^{\prime}$ UTR of amyloid$\beta$ protein precursor converting enzyme (BACE1) protein mRNA, and exert regulatory effects on BACE1 protein expression in cultured neuronal cells [15]. Exosomes derived from AD and Down syndrome blood samples were shown to contain lower $A \beta_{42}$ and increased p-tau [16] reflecting the 'classic' AD cerebrospinal fluid (CSF) biomarker profile [17], suggesting that exosomal miRNA expression profiling may provide further information on
AD-relevant pathophysiological mechanisms. Other miRNAs with diagnostic biomarker potential include miR-320a and miR-204-5p, where upregulation of miR-320a [7] and downregulation of miR204-5p [9] have been reported in the CSF of patients with $\mathrm{AD}$ and FTD respectively. CSF miR-320a was shown to be upregulated in patients with young-onset Alzheimer's disease (YOAD) [7]. CSF exosomal miR-204-5p was reported to be downregulated in symptomatic compared to presymptomatic FTD mutation carriers [9], with target mRNAs enriched in frontal and temporal lobes. However, it is not known whether miR-204-5p is dysregulated in AD. Expression levels of serum circulating miR-328-3p were also substantially downregulated in autism spectrum disorder patients compared to healthy controls [18]. In our miRNA-sequencing work conducted in another cohort, miR-328-3p was shown to be downregulated in the peripheral blood mononuclear cell (PBMC) of young onset $\mathrm{AD}$ cases compared to controls (Chia et al., manuscript under review).

Differentiating AD and FTD are often challenging in the early stages of disease, with overlapping symptoms. To determine if specific exosomal miRNAs can be validated as reliable biomarkers for $\mathrm{AD}$ and/or FTD, we investigated the expression of three exosomal miRNAs (miR-320a, miR-328-3p, and miR-204-5p) isolated from the CSF of patients with YOAD (defined as occurring before the age of 65 years), and FTD.

Currently, miRNA studies produce inconsistent results and are rarely validated, which limit their potential to be established as clinical biomarkers. Standardized and consistent methods can help to limit technical bias and inconsistent results. Hence, we sought to address this gap by: 1) validating miR204-5p as a marker for sporadic FTD and AD; 2) validating miR-320a as a biomarker for YOAD; and 3) investigating miR328-3p as a novel marker for $A D$, using the same methodology on isolation of CSF exosomes and miRNA measurement as previous studies $[7,9]$. 


\section{MATERIALS AND METHODS}

\section{Study participants}

Participants aged less than 65 years diagnosed with dementia were recruited from the memory clinics at the National Neuroscience Institute, Singapore between 2015 and 2018. This study was approved by the SingHealth Institutional Ethics Review Board and written informed consent was obtained from each participant prior to recruitment into the study. All patients underwent neurological examination, neuropsychological assessment (global cognition was measured using the Mini-Mental State Examination (MMSE) and Montreal Cognitive Assessment (MoCA) tools), brain magnetic resonance imaging (MRI), and met consensus diagnostic criteria for AD [17] and sporadic behavioral variant FTD (bvFTD) [19] as determined by cognitive neurologists. All healthy controls (HCs) were free of significant neurological, psychiatric, or systemic disease. Patients with other neurodegenerative diseases such as Parkinson's disease, with psychiatric comorbidities, as well as a history of alcohol or drug abuse were excluded.

\section{CSF collection}

All CSF samples were collected by lumbar puncture in polypropylene tubes, followed by centrifugation at $2,000 \times \mathrm{g} 10 \mathrm{~min}$. CSF samples were aliquoted into polypropylene tubes and stored at $-80^{\circ} \mathrm{C}$ until further analysis. The concentration of $\mathrm{CSF} A \beta_{42}$, $\mathrm{p}$-tau, and t-tau were determined by INNOTEST ELISAs (Fujirebio), according to the manufacturer's instructions.

\section{Isolation of exosome and miRNA}

Methods used to isolate exosome from CSF and measure miRNA expression have been described before [9] with slight modifications. Briefly, a fixed volume of $0.5 \mathrm{ml}$ CSF samples was used to isolate exosomes from each participant using miRCURY Exosome Cell/Urine/CSF Kit (Qiagen) according to the manufacturer's instructions. CSF samples were incubated with Precipitation Buffer at $4^{\circ} \mathrm{C}$ for overnight and spun for $30 \mathrm{~min}$ at $10000 \times \mathrm{g}$ at $20^{\circ} \mathrm{C}$ to pellet the exosome, which was then resuspended with $200 \mu \mathrm{l}$ Resuspension Buffer. Exosomes isolated from CSF samples were subjected to cryo-transmission electron microscopy analysis to confirm the presence of vesicular structures and nanoparticle tracking analysis to obtain concentrations and size-distribution of exosome like-vesicles from the isolated samples.

Exosomal RNA was isolated using miRNeasy Micro Kit (Qiagen), following manufacturer's protocol. Complementary DNA (cDNA) was synthesized using miRCURY LNA RT kit (Qiagen). RNA Spikein Mix was added during the first step of RNA isolation and cDNA synthesis. Quantitative realtime PCR (qRT-PCR) was performed to measure expression levels of miR-204-5p, miR-320a, and miR-328-3p using miRCURY LNA primer sets and SYBR Green assay (Qiagen). MiRNA expressions were calculated relative to $\mathrm{HCs}$ using the $2{ }_{-} \Delta \Delta \mathrm{Ct}$ method (Livak Methods 2001) with $\Delta \mathrm{Ct}=\mathrm{Ct}_{\text {miRNA }}$ $-\mathrm{Ct}_{\text {reference }}$ and $\Delta \Delta \mathrm{Ct}=\Delta \mathrm{Ct}_{\text {patient }}-\Delta \mathrm{Ct}_{\mathrm{HC}}$. pool. UniSp6 spike-in was used as a reference for normalization.

\section{Cryo-transmission electron microscopy (Cryo-TEM) analysis}

For cryo-TEM, $4 \mu \mathrm{L}$ of exosome preparations were directly adsorbed onto lacey carbon grids (Quantifoil, Germany) and plunged into liquid ethane, using an FEI Vitrobot Mark 3 (FEI Company, The Netherlands). Grids were blotted at $100 \%$ humidity at $4{ }^{\circ} \mathrm{C}$ for about 3-4 s. Frozen/vitrified samples were imaged using Tecnai T12 Transmission Electron Microscope (FEI Company) operating at an acceleration voltage of $120 \mathrm{kV}$. Images were taken at 30,000x magnification, (approximate dose of 13.6 electrons/Å2), using an FEI Eagle 4k CCD (FEI Company), and Serial EM image acquisition software.

\section{NTA measurement with Nanosight NS300 (Malvern, UK)}

All samples were diluted in PBS to a final volume of $1 \mathrm{ml}$. Ideal measurement concentrations were found by pre-testing the ideal particle per frame value (20-100 particles/frame). Following settings were set according to the manufacturer's software manual (NanoSight NS300 User Manual): camera level was increased until all particles were distinctly visible not exceeding a particle signal saturation over $20 \%$. The ideal detection threshold was determined to include as many particles as possible with the restrictions that 10-1000 red crosses were counted while only $<10 \%$ were not associated with distinct particles. Blue cross count was limited to 5. Autofocus was adjusted so that indistinct particles were avoided. For each measurement, images and videos were captured. 
Table 1

Demographic and clinical characteristics of participants

\begin{tabular}{lcccc}
\hline Parameter & $\mathrm{HC}$ & $\mathrm{AD}$ & FTD & $p$ \\
\hline Subjects, $n$ & 8 & 28 & 12 & \\
Age, y & $54.6 \pm 7.6$ & $58.2 \pm 4.8$ & $57.4 \pm 6.2$ & 0.286 \\
Male sex, $\mathrm{n}(\%)$ & $7(88)$ & $12(43)$ & $5(42)$ & 0.067 \\
Education, y & $15.0 \pm 4.5$ & $11.7 \pm 3.8$ & $12.6 \pm 3.2$ & 0.235 \\
MMSE & $28.0 \pm 1.8$ & $18.7 \pm 6.9$ & $24.0 \pm 5.6$ & 0.002 \\
MoCA & $27.6 \pm 2.6$ & $16.3 \pm 6.6$ & $21.8 \pm 6.5$ & $<0.001$ \\
CSF A $\beta_{42}, \mathrm{pg} / \mathrm{ml}$ & $1073.3 \pm 358.8$ & $694.7 \pm 366.8$ & $1169.0 \pm 1006.6$ & 0.052 \\
CSF t-tau, pg/ml & $231.5 \pm 47.3$ & $757.5 \pm 519.9$ & $398.1 \pm 202.8$ & 0.003 \\
CSF p-tau, pg/ml & $39.3 \pm 14.3$ & $91.5 \pm 51.5$ & $52.5 \pm 21.9$ & 0.003 \\
\hline
\end{tabular}

Continuous variables reported as mean \pm standard deviation; Categorical variables reported as $\mathrm{n}(\%)$. MMSE, Mini-Mental State Examination; MoCA, Montreal Cognitive Assessment; $A \beta_{42}$, amyloid- $\beta$ 42; t-tau, total tau; p-tau, phosphorylated tau 181.

After capture, the videos have been analyzed by the in-build NanoSight Software NTA 3.1 Build 3.1.46 with a detection threshold of 5. Hardware: embedded laser: $45 \mathrm{~mW}$ at $488 \mathrm{~nm}$; camera: sCMOS. The number of completed tracks in NTA measurements was always greater than the proposed minimum of 1000 in order to minimize data skewing based on single large particles. There was no significant difference in concentration $(p=0.632)$ and size of isolated exosomes $(p=0.604)$ across disease groups. The mean \pm SD concentration of isolated exosomes in $\mathrm{HCs}, \mathrm{AD}$, and FTD were $2.5 \pm 1.8,3.3 \pm 1.5$, and $3.4 \pm 2.1$, respectively. The mean $\pm \mathrm{SD}$ size distribution of isolated exosomes in HCs, AD, and FTD were $159.5 \pm 38.0$, $176.1 \pm 35.1$, and $167.5 \pm 36.5$, respectively.

\section{Characterization of extracellular vesicles}

Characterization of isolated exosomes using cryoTEM suggested that these vesicles predominantly contained double-walled lipid membrane layers (Fig. 3A, B). Particle analysis using NanoSight measurements (Fig. 3C) suggested that the large population of exosomes (e.g., $120 \pm 0.7 \%$ ) fell within the size range of $30-350 \mathrm{~nm}$ with average vesicle size determined to be $111 \pm 3.07 \mathrm{~nm}[20,21]$. The morphological and physical characteristics determined using these techniques corroborate with previous evidence [22, 23], suggesting that these nanovesicles are probably of exosomal origin and were hence used for further experiments on miRNA isolation and analysis.

\section{Target prediction and pathway enrichment analysis}

miR-328-3p was selected and subjected to target prediction using two online databases: TargetScan (http://www.targetscan.org/) and miRDB (http:// www.mirdb.org/) to predict miRNA targets. TargetScan predicted 211 targets and miRDB predicted 279 targets for miR-328-3p (Supplementary Material). Only 69 common genes predicted by both databases were considered. Gene-annotation enrichment analyses of the target genes were performed using DAVID Bioinformatic Resources v6.8 (https://david.ncifcrf.gov/) to identify biological processes, cellular components, molecular function, and pathway annotation of the common genes [24]. A $p<0.05$ value was considered significant for enrichment analyses.

\section{Statistical analysis}

Continuous variables were compared across the disease groups (HC, $\mathrm{AD}$, and FTD) using one-way ANOVA while categorical variables were compared using Chi-square test. Normality assumption was assessed by visual inspection via PP plots on the residuals of the ANOVA test. No major deviation from normality assumption was observed. Significant ANOVA results were followed by least significant difference (LSD) and Bonferroni's post hoc tests. Correlations between clinical data and miRNA expression levels were calculated using Pearson's correlation. The diagnostic accuracy of miRNAs was assessed with the receiving operating characteristic (ROC) curve analysis. $p \leq 0.05$ was considered statistically significant. All statistics were done using SPSS version 23 (IBM).

\section{RESULTS}

A total of 48 participants were included in this study: $8 \mathrm{HCs}, 12 \mathrm{FTD}$, and $28 \mathrm{AD}$ cases. The demographics and clinical characteristics of our study cohort are presented in Table 1 . There was 

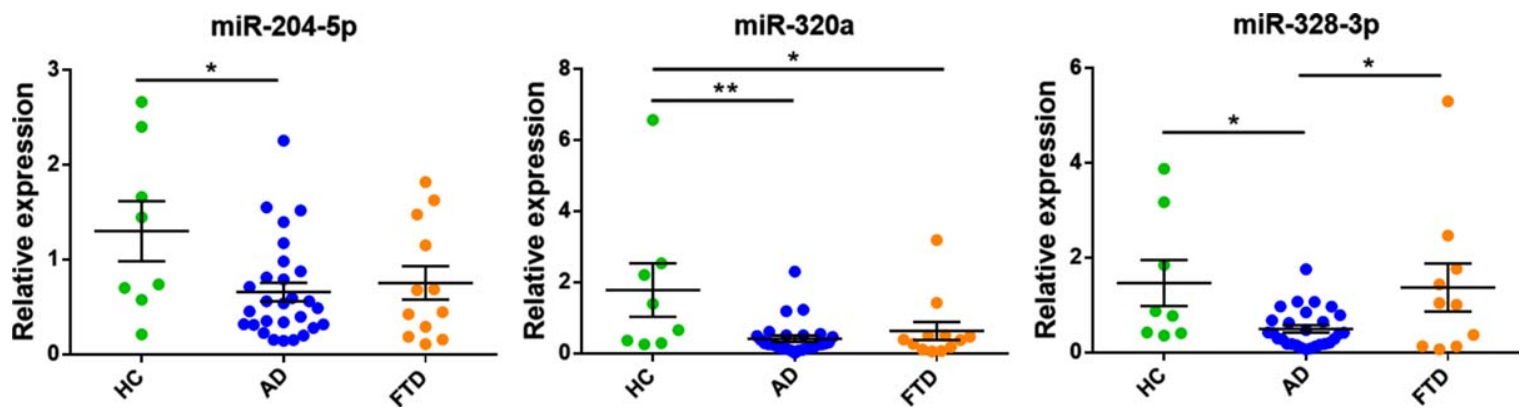

Fig. 1. Relative expression of miR-204-5p, miR-320a, and miR-328-3p in exosomes from CSF of young-onset AD, FTD, and HCs. Data are presented as the mean \pm SEM. One-way ANOVA with Bonferroni post hoc test was used to compare the differences between disease groups. ${ }^{*} p \leq 0.05 ;{ }^{* *} p \leq 0.01$.
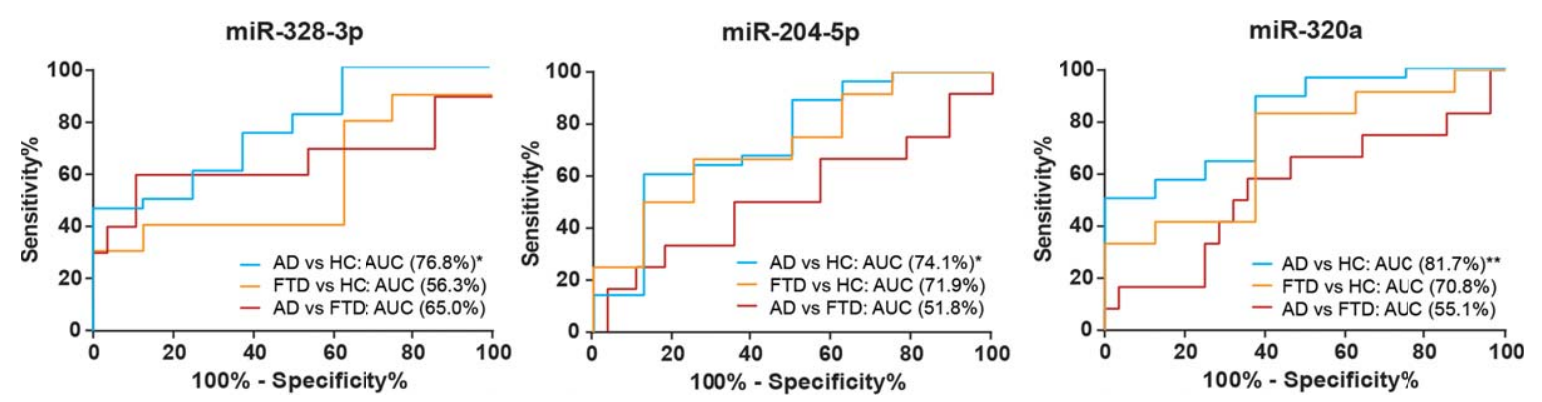

Fig. 2. Receiving operating characteristic (ROC) curves of CSF exosomal miR-328-3p, miR-204-5p, and miR-320a levels between AD, FTD and HC. ${ }^{*} p<0.05 ;{ }^{* *} p<0.01$.

no significant association between all three miRNAs (miR-204-5p, miR-320a, and miR-328-3p) and age, gender, or education $(p>0.05)$. Within patient groups, none of the miRNAs showed significant correlation with global cognitive scores as measured by the MMSE and MoCA $(p>0.05)$.

The relative expression of CSF exosomal miR204-5p, miR-320a, and miR-328-3p differed across the three groups $(p=0.041, p=0.006$, and $p=0.016$, respectively; Fig. 1). Post-hoc analysis revealed:

(i) Lower miR-204-5p expression levels in $\mathrm{AD}$ $(p=0.012$, Bonferroni corrected $p=0.036)$ and FTD $(p=0.057$, Bonferroni corrected $p=0.170)$ compared to HCs. There was no difference in miR-204-5p levels between AD and FTD $(p=0.654)$. No significant association with CSF A $\beta_{42}$, t-tau, and p-tau levels was found.

(ii) The relative expression of miR-320a was significantly lower in $\mathrm{AD}(p=0.002$, Bonferroni corrected $p=0.005)$ and FTD cases $(p=0.016$, Bonferroni corrected $p=0.049)$ compared to $\mathrm{HCs}$, but no difference was found between $\mathrm{AD}$ and FTD groups $(p=0.537)$. There was no significant association with $\mathrm{CSF} A \beta_{42}$, t-tau, and p-tau levels.

(iii) $\mathrm{miR}-328-3 p$ expression levels were lowest in AD cases, compared to HCs ( $p=0.016$, Bonferroni corrected $p=0.049)$ and FTD $(p=0.018$, Bonferroni corrected $p=0.054)$. Within patient groups, lower miR-328-3p expression correlated significantly with lower CSF A $\beta_{42}$ levels $(r=0.359, p=0.029)$, but not with t-tau and p-tau. Additionally, we found that lower miR328-3p levels could differentiate AD from FTD and HCs with AUC: 0.702 , 95\% CI: 0.5340.870 . Results of ROC analysis are shown in Fig. 2.

Given that the result of lower miR-328-3p expression levels in $\mathrm{AD}$ was consistent with our earlier findings in our miRNA-sequencing discovery cohort and its ability to differentiate AD from FTD and HCs, we sought to identify the possible downstream pathway enriched by the target mRNAs of miR-328-3p. Using two computational prediction databases (TargetScan and miRDB), we found 69 

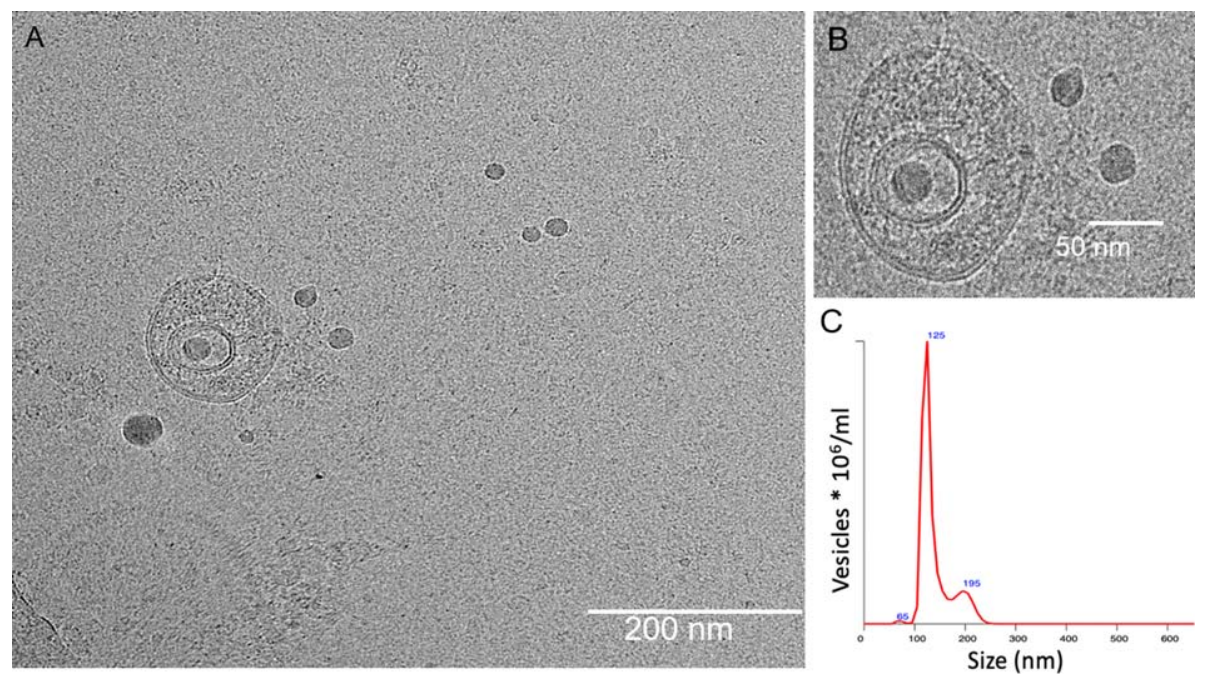

Fig. 3. Cryo-transmission electron microscopy images of exosomes isolated from CSF sample. (A) Double-walled membrane vesicles identified from CSF sample. (B) High-resolution micrograph of the double-walled membrane vesicle. Scale bar (A) $200 \mathrm{~nm}$ and (B) $50 \mathrm{~nm}$, respectively. (C) Size distribution and concentration of isolated exosomes evaluated by Nanosight.

overlapping mRNAs targeted by miR-328-3p (Supplementary Material). These target mRNAs were then subjected to Gene-annotation enrichment analyses using DAVID Bioinformatic Resources (v6.8) to identify the functional role of miRNA targets (see the Supplementary Material for more details). Gene Ontology (GO) analysis revealed enrichment in biological processes involving negative regulation of translation, neural tube formation, regulation of neural precursor cell proliferation, and insulin receptor signaling pathway. The molecular functions of target genes included protein binding, beta-catenin binding, sequence-specific DNA binding, and translation repressor activity. Cellular components that were enriched include early endosome, cytoplasm, cytosol, and membrane. Kyoto Encyclopedia of Genes and Genomes (KEGG) pathway analysis identified potential targets of miR-328-3p implicated in the AMP-activated protein kinase (AMPK) signaling pathway, acute myeloid leukemia, mTOR signaling pathway, and choline metabolism in cancer, with the most significantly enriched pathway being the AMPK signaling pathway. The target genes mapped in this pathway include CPT1A, EIF4EBP1, TSC1, and $P P P 2 R 5 D$.

\section{DISCUSSION}

In our search for viable diagnostic biomarkers, we sought to validate three CSF exosomal miRNA targets that have previously shown to be altered in dementia compared to HCs. We showed that CSF exosomal miR-320a and miR-204-5p levels were markers for dementia, being significantly downregulated in AD and FTD compared to HCs but could not differentiate AD from FTD. CSF exosomal miR328-3p, however, was downregulated in AD patients and could differentiate $\mathrm{AD}$ from $\mathrm{HCs}$, and from FTD.

Downregulation of miR-320a has been reported in the serum of $\mathrm{AD}$ and sporadic bvFTD patients compared to HC, where miR-320a and miR-320b showed good diagnostic accuracy at discriminating bvFTD and AD cases from controls [25]. In our study, we similarly found that miR-320a could discriminate $\mathrm{AD}$ and FTD from HCs, but not between diagnostic groups. Overall, our study provides further validation of downregulated miR-320a as a marker for dementia.

CSF exosomal miR-204-5p has had conflicting results where it was significantly downregulated in symptomatic genetic FTD mutation carriers compared to presymptomatic carriers and non-carriers but not in a cohort of sporadic FTD cases compared to $\mathrm{HC}$ [9]. We found downregulation of miR-204-5p in our sporadic FTD cases compared to controls, but this downregulation was also seen in our AD cases and may reflect underlying symptomatic neurodegeneration rather than specific disease pathophysiology. Moreover, target genes of miR-204-5p were previously reported to be highly expressed in frontal and temporal lobes [9]—regions that are commonly affected in FTD and some variants of YOAD. These 
findings provide opportunity for miR-204-5p to be validated in further studies.

Importantly, we report for the first time that CSF exosomal miR-328-3p was significantly downregulated in AD compared to FTD and HC. This result was in accordance with and validated the findings from our previous miRNA-sequencing analysis on peripheral blood from a separate AD cohort (Chia et al., under review). Moreover, lower miR-328-3p expression showed significant association with lower CSF A $\beta_{42}$ levels, a result that can be supported by findings of lower miR-328 expression levels in the hippocampus of $\mathrm{APP}_{\text {Swe }} / \mathrm{PS} 1$ mice [15], correlating with higher BACE1 protein expression, which was reported to be increased in the brain of AD patients [26]. Serum miR-328-3p expression has also been reported to be downregulated in children with autism spectrum disorder [18].

Pathway analysis suggests that the target genes of miR-328-3p are involved in the AMPK signaling pathway, which has been reported to be affected in $\mathrm{AD}$ [27]. The target genes mapped in this pathway include CPTIA, EIF4EBP1, TSC1, and PPP2R5D. AMPK is one of the central regulators of cellular and organismal metabolism in eukaryotes, regulating growth and reprogramming metabolism, and involved in cellular processes such as autophagy and cell polarity [28]. In AD, the AMPK signaling pathway was proposed to be involved in the degradation of amyloid- $\beta$ peptides and in tau phosphorylation [27], the two key hallmarks of AD. In vivo studies have shown that AMPK hyperactivation leads to reduced synaptic integrity [29, 30]. Decreased mRNA expression of AMPK was reported in the hippocampus of aging and transgenic AD mice and impaired AMPK function in mice displayed AD-like tau hyperphosphorylation and memory impairment [31]. The target genes mapped in this pathway include CPTIA, EIF4EBPI, TSC1, and PPP2R5D. Carnitine Palmitoyltransferase $1 \mathrm{~A}(C P T 1 A)$ resides in the outer mitochondrial membrane and is involved in fatty acid oxidation, a process that is affected in $\mathrm{AD}$ [32]. Reduced CPT activity in AD has been suggested by previous studies $[33,34]$. Eukaryotic translation initiation factor 4E-binding protein 1 (EIF4EBP1) functions as a translation repressor protein, and phosphorylated EIF4EBP1 levels have been reported to be significantly increased in $\mathrm{AD}$ brains compared to controls [35], while increased levels have been found in the CSF and plasma of AD patients [36]. Interestingly, tuberous sclerosis protein 1 (TSC1) has been genetically linked with AD, while loss of TSC1 is associated with tauopathy in in vitro and in vivo studies [37, 38]. Protein Phosphatase 2 Regulatory Subunit B'Delta (PPP2R5D) has also been associated with tau hyperphosphorylation $[39,40]$ and studies have reported decreased PPP2R5D expression and activity in the AD hippocampus [41, 42]. Taken together, downregulation of miR-328-3p could contribute to AD pathology through dysregulation of the AMPK pathway and its target genes, potentially affecting downstream processes that may result in abnormal amyloid- $\beta$, tau, and fatty acid metabolism. Experimental validation of these molecules in $\mathrm{AD}$ is warranted in future studies.

Overall, we validated the downregulation of CSF exosomal miR-320a and miR-204-5p in AD and FTD compared to HCs, while we also show the potential of miR-328-3p as a novel diagnostic biomarker for AD. Future studies with a larger cohort of patients and HCs are needed to confirm these key findings.

\section{ACKNOWLEDGMENTS}

We would like to thank all subjects for their participation in this study. This research was supported by the Singapore National Medical Research Council (NMRC/CIRG/1416/2015 and MOH-TA18 may-0003).

\section{CONFLICT OF INTEREST}

The authors have no conflict of interest to report.

\section{SUPPLEMENTARY MATERIAL}

The supplementary material is available in the electronic version of this article: https://dx.doi.org/ 10.3233/ADR210311.

\section{REFERENCES}

[1] Eulalio A, Huntzinger E, Izaurralde E (2008) Getting to the root of miRNA-mediated gene silencing. Cell 132, 9-14.

[2] Molasy M, Walczak A, Szaflik J, Szaflik JP, Majsterek I (2017) microRNAs in glaucoma and neurodegenerative diseases. J Hum Genet 62, 105-112.

[3] Valadi H, Ekström K, Bossios A, Sjöstrand M, Lee JJ, Lötvall JO (2007) Exosome-mediated transfer of mRNAs and microRNAs is a novel mechanism of genetic exchange between cells. Nat Cell Biol 9, 654-659.

[4] Zhang Y, Liu Y, Liu H, Tang WH (2019) Exosomes: biogenesis, biologic function and clinical potential. Cell Biosci 9, 19 .

[5] Sørensen SS, Nygaard A-B, Christensen T (2016) miRNA expression profiles in cerebrospinal fluid and blood of 
patients with Alzheimer's disease and other types of dementia - an exploratory study. Transl Neurodegener 5,6 .

[6] Lusardi TA, Phillips JI, Wiedrick JT, Harrington CA, Lind B, Lapidus JA, Quinn JF, Saugstad JA (2017) microRNAs in human cerebrospinal fluid as biomarkers for Alzheimer's disease. J Alzheimers Dis 55, 1223-1233.

[7] McKeever PM, Schneider R, Taghdiri F, Weichert A, Multani N, Brown RA, Boxer AL, Karydas A, Miller B, Robertson J, Tartaglia MC (2018) MicroRNA expression levels are altered in the cerebrospinal fluid of patients with young-onset Alzheimer's disease. Mol Neurobiol 55, 88268841.

[8] Hajjri SN, Sadigh-Eteghad S, Mehrpour M, Moradi F, Shanehbandi D, Mehdizadeh M (2020) Beta-amyloiddependent miRNAs as circulating biomarkers in Alzheimer's disease: a preliminary report. J Mol Neurosci 70, 871-877.

[9] Schneider R, McKeever P, Kim T, Graff C, van Swieten JC, Karydas A, Boxer A, Rosen H, Miller BL, Laforce Jr R, Galimberti D, Masellis M, Borroni B, Zhang Z, Zinman L, Rohrer JD, Tartaglia MC, Robertson J (2018) Downregulation of exosomal miR-204-5p and miR-632 as a biomarker for FTD: a GENFI study. J Neurol Neurosurg Psychiatry 89, 851-858.

[10] Grasso M, Piscopo P, Talarico G, Ricci L, Crestini A, Tosto G, Gasparini M, Bruno G, Denti MA, Confaloni A (2019) Plasma microRNA profiling distinguishes patients with frontotemporal dementia from healthy subjects. $\mathrm{Neu}$ robiol Aging 84, 240.e1-240.e12.

[11] Cheng L, Sharples RA, Scicluna BJ, Hill AF (2014) Exosomes provide a protective and enriched source of miRNA for biomarker profiling compared to intracellular and cellfree blood. J Extracell Vesicles 3, 23743.

[12] Boukouris S, Mathivanan S (2015) Exosomes in bodily fluids are a highly stable resource of disease biomarkers. Prot Clin Appl 9, 358-367.

[13] Rajendran L, Honsho M, Zahn TR, Keller P, Geiger KD, Verkade P, Simons K (2006) Alzheimer's disease betaamyloid peptides are released in association with exosomes. Proc Natl Acad Sci U S A 103, 11172-11177.

[14] Yuyama K, Sun H, Usuki S, Sakai S, Hanamatsu H, Mioka T, Kimura N, Okada M, Tahara H, Furukawa J, Fujitani N, Shinohara Y, Igarashi Y (2015) A potential function for neuronal exosomes: sequestering intracerebral amyloid- $\beta$ peptide. FEBS Lett 589, 84-88.

[15] Boissonneault V, Plante I, Rivest S, Provost P (2009) MicroRNA-298 and MicroRNA-328 regulate expression of mouse $\beta$-amyloid precursor protein-converting enzyme 1 . $J$ Biol Chem 284, 1971-1981.

[16] Hamlett ED, Goetzl EJ, Ledreux A, Vasilevko V, Boger HA, LaRosa A, Clark D, Carroll SL, Carmona-Iragui M, Fortea J, Mufson EJ, Sabbagh M, Mohammed AH, Hartley D, Doran E, Lott IT, Granholm A-C (2017) Neuronal exosomes reveal Alzheimer's disease biomarkers in Down syndrome. Alzheimers Dement 13, 541-549.

[17] McKhann GM, Knopman DS, Chertkow H, Hyman BT, Jack CR, Kawas CH, Klunk WE, Koroshetz WJ, Manly JJ, Mayeux R, Mohs RC, Morris JC, Rossor MN, Scheltens P, Carrillo MC, Thies B, Weintraub S, Phelps $\mathrm{CH}$ (2011) The diagnosis of dementia due to Alzheimer's disease: recommendations from the National Institute on Aging-Alzheimer's Association workgroups on diagnostic guidelines for Alzheimer's disease. Alzheimers Dement 7, 263-269.
[18] Popov N, Minchev D, Naydenov M, Minkov I, Vachev T (2018) Investigation of circulating serum MicroRNA-328$3 p$ and MicroRNA-3135a expression as promising novel biomarkers for autism spectrum disorder. Balkan J Med Genet 21, 5-12.

[19] Rascovsky K, Hodges JR, Knopman D, Mendez MF, Kramer JH, Neuhaus J, van Swieten JC, Seelaar H, Dopper EGP, Onyike CU, Hillis AE, Josephs KA, Boeve BF, Kertesz A, Seeley WW, Rankin KP, Johnson JK, Gorno-Tempini ML, Rosen H, Prioleau-Latham CE, Lee A, Kipps CM, Lillo P, Piguet O, Rohrer JD, Rossor MN, Warren JD, Fox NC, Galasko D, Salmon DP, Black SE, Mesulam M, Weintraub S, Dickerson BC, Diehl-Schmid J, Pasquier F, Deramecourt V, Lebert F, Pijnenburg Y, Chow TW, Manes F, Grafman J, Cappa SF, Freedman M, Grossman M, Miller BL (2011) Sensitivity of revised diagnostic criteria for the behavioural variant of frontotemporal dementia. Brain 134, 24562477.

[20] Lane RE, Korbie D, Anderson W, Vaidyanathan R, Trau M (2015) Analysis of exosome purification methods using a model liposome system and tunable-resistive pulse sensing. Sci Rep 5, 7639.

[21] Vaidyanathan R, Naghibosadat M, Rauf S, Korbie D, Carrascosa LG, Shiddiky MJA, Trau M (2014) Detecting exosomes specifically: a multiplexed device based on alternating current electrohydrodynamic induced nanoshearing. Anal Chem 86, 11125-11132.

[22] Coleman BM, Hanssen E, Lawson VA, Hill AF (2012) Prion-infected cells regulate the release of exosomes with distinct ultrastructural features. FASEB $j$ 26, 4160-4173.

[23] de Vrij J, Maas SL, van Nispen M, Sena-Esteves M, Limpens RW, Koster AJ, Leenstra S, Lamfers ML, Broekman ML (2013) Quantification of nanosized extracellular membrane vesicles with scanning ion occlusion sensing. Nanomedicine 8, 1443-1458.

[24] Huang DW, Sherman BT, Lempicki RA (2009) Systematic and integrative analysis of large gene lists using DAVID bioinformatics resources. Nat Protoc 4, 44-57.

[25] Denk J, Oberhauser F, Kornhuber J, Wiltfang J, Fassbender K, Schroeter ML, Volk AE, Diehl-Schmid J, Prudlo J, Danek A, Landwehrmeyer B, Lauer M, Otto M, Jahn H, FTLDc study group (2018) Specific serum and CSF microRNA profiles distinguish sporadic behavioural variant of frontotemporal dementia compared with Alzheimer patients and cognitively healthy controls. PLoS One 13, e0197329.

[26] Holsinger RMD, McLean CA, Beyreuther K, Masters CL, Evin G (2002) Increased expression of the amyloid precursor $\beta$-secretase in Alzheimer's disease. Ann Neurol 51, 783-786.

[27] Assefa BT, Tafere GG, Wondafrash DZ, Gidey MT (2020) The bewildering effect of AMPK activators in Alzheimer's disease: review of the current evidence. Biomed Res Int 2020, 9895121.

[28] Mihaylova MM, Shaw RJ (2011) The AMPK signalling pathway coordinates cell growth, autophagy and metabreakbolism. Nat Cell Biol 13, 1016-1023.

[29] Domise M, Sauvé F, Didier S, Caillerez R, Bégard S, Carrier S, Colin M, Marinangeli C, Buée L, Vingtdeux V (2019) Neuronal AMP-activated protein kinase hyper-activation induces synaptic loss by an autophagy-mediated process. Cell Death Dis 10, 221.

[30] Mairet-Coello G, Courchet J, Pieraut S, Courchet V, Maximov A, Polleux F (2013) The CAMKK2-AMPK kinase pathway mediates the synaptotoxic effects of $A \beta$ oligomers through tau phosphorylation. Neuron 78, 94-108. 
[31] Wang L, Li N, Shi F-X, Xu W-Q, Cao Y, Lei Y, Wang J-Z, Tian Q, Zhou X-W (2020) Upregulation of AMPK ameliorates Alzheimer's disease-like tau pathology and memory impairment. Mol Neurobiol 57, 3349-3361.

[32] Montine TJ, Morrow JD (2005) Fatty acid oxidation in the pathogenesis of Alzheimer's disease. Am J Pathol 166, 1283-1289.

[33] Cristofano A, Sapere N, La Marca G, Angiolillo A, Vitale M, Corbi G, Scapagnini G, Intrieri M, Russo C, Corso G, Di Costanzo A (2016) Serum levels of Acyl-carnitines along the continuum from normal to Alzheimer's dementia. PLoS One 11, e0155694.

[34] Virmani A, Pinto L, Bauermann O, Zerelli S, Diedenhofen A, Binienda ZK, Ali SF, van der Leij FR (2015) The carnitine palmitoyl transferase (CPT) system and possible relevance for neuropsychiatric and neurological conditions. Mol Neurobiol 52, 826-836.

[35] Li X, Alafuzoff I, Soininen H, Winblad B, Pei J-J (2005) Levels of mTOR and its downstream targets 4E-BP1, eEF2, and eEF2 kinase in relationships with tau in Alzheimer's disease brain: Abnormal translation control in Alzheimer's disease. FEBS J 272, 4211-4220.

[36] Whelan CD, Mattsson N, Nagle MW, Vijayaraghavan S, Hyde C, Janelidze S, Stomrud E, Lee J, Fitz L, Samad TA, Ramaswamy G, Margolin RA, Malarstig A, Hansson O (2019) Multiplex proteomics identifies novel CSF and plasma biomarkers of early Alzheimer's disease. Acta Neuropathol Commun 7, 169.

[37] Alquezar C, Schoch KM, Geier EG, Ramos EM, Scrivo A, Li K, Argouarch AR, Mlynarski EE, Dombroski B, Yokoyama JS, Cuervo AM, Burlingame AL, Schellenberg GD, Miller TM, Miller BL, Kao AW (2020) TSC1 loss-offunction increases risk for tauopathy by inducing tau acetylation and preventing autophagy-mediated tau clearance. bioRxiv 2020.11.08.371922; doi: https://doi.org/10.1101/ 2020.11.08.371922.
[38] Sanchez CG, Acker CM, Gray A, Varadarajan M, Song C, Cochran NR, Paula S, Lindeman A, An S, McAllister G, Alford J, Reece-Hoyes J, Russ C, Craig L, Capre K, Doherty C, Hoffman GR, Luchansky SJ, Polydoro M, Dolmetsch R, Elwood F (2021) Genome-wide CRISPR screen identifies protein pathways modulating tau protein levels in neurons. Commun Biol 4, 736.

[39] Yu UY, Yoo BC, Ahn J-H (2014) Regulatory B subunits of protein phosphatase $2 \mathrm{~A}$ are involved in site-specific regulation of tau protein phosphorylation. Korean J Physiol Pharmacol 18, 155.

[40] Louis JV, Martens E, Borghgraef P, Lambrecht C, Sents W, Longin S, Zwaenepoel K, Pijnenborg R, Landrieu I, Lippens G, Ledermann B, Gotz J, Van Leuven F, Goris J, Janssens V (2011) Mice lacking phosphatase PP2A subunit PR61/B' (Ppp2r5d) develop spatially restricted tauopathy by deregulation of CDK5 and GSK3. Proc Natl Acad Sci U S A 108, 6957-6962.

[41] Sontag E, Luangpirom A, Hladik C, Mudrak I, Ogris E, Speciale S, White CL (2004) Altered expression levels of the protein phosphatase $2 \mathrm{~A} \mathrm{AB} \alpha \mathrm{C}$ enzyme are associated with Alzheimer disease pathology. J Neuropathol Exp Neurol 63, 287-301.

[42] Vogelsberg-Ragaglia V, Schuck T, Trojanowski JQ, Lee VM-Y (2001) PP2A mRNA expression is quantitatively decreased in Alzheimer's disease hippocampus. Exp Neurol 168, 402-412. 